\title{
Conductance oscillations of core-shell nanowires in transversal magnetic fields
}

\author{
Andrei Manolescu, ${ }^{1, *}$ George Alexandru Nemnes, ${ }^{2,3}$ Anna Sitek, ${ }^{4,5}$ Tomas Orn Rosdahl, ${ }^{6}$ \\ Sigurdur Ingi Erlingsson, ${ }^{1}$ and Vidar Gudmundsson ${ }^{4}$ \\ ${ }^{1}$ School of Science and Engineering, Reykjavik University, Menntavegur 1, IS-101 Reykjavik, Iceland \\ ${ }^{2}$ University of Bucharest, Faculty of Physics, MDEO Research Center, 077125 Magurele-Ilfov, Romania \\ ${ }^{3}$ Horia Hulubei National Institute for Physics and Nuclear Engineering, 077126 Magurele-Ilfov, Romania \\ ${ }^{4}$ Science Institute, University of Iceland, Dunhaga 3, IS-107 Reykjavik, Iceland \\ ${ }^{5}$ Department of Theoretical Physics, Faculty of Fundamental Problems of Technology, \\ Wroclaw University of Technology, 50-370 Wroclaw, Poland \\ ${ }^{6}$ Kavli Institute of Nanoscience, Delft University of Technology, 2600 GA Delft, The Netherlands \\ (Received 7 January 2016; revised manuscript received 4 May 2016; published 31 May 2016)
}

\begin{abstract}
We analyze theoretically electronic transport through a core-shell nanowire in the presence of a transversal magnetic field. We calculate the conductance for a variable coupling between the nanowire and the attached leads and show how the snaking states, which are low-energy states localized along the lines of the vanishing radial component of the magnetic field, manifest their existence. In the strong-coupling regime they induce flux periodic, Aharonov-Bohm-like, conductance oscillations, which, by decreasing the coupling to the leads, evolve into well-resolved peaks. The flux periodic oscillations arise due to interference of the snaking states, which is a consequence of backscattering at either the contacts with leads or magnetic or potential barriers in the wire.
\end{abstract}

DOI: 10.1103/PhysRevB.93.205445

\section{INTRODUCTION}

Design and technological realization of quantum nanodevices requires nanoscale systems of well-defined and controllable properties. Recently, tubular semiconductor structures turned out to be promising building blocks of such appliances. Nanotubes of very narrow but finite thickness may be achieved in a few different ways. In the case of quantum wires built of narrow-gap materials surface states may induce Fermi-level pinning above the conduction-band edge, which results in accumulation of electrons in the vicinity of the surface [1]. Nowadays it has become feasible to combine two (or even more) different materials into one vertical structure, i.e., core-shell nanowires (CSNs). This provides a possibility to achieve thin tubular shells surrounding a core nanowire or other shells. One of the advantages of these systems is a possibility to establish band alignment through the thicknesses of the components [2-4] and thus grow structures in which electrons are confined only in narrow shell areas [5,6]. Moreover, the core part may be etched such that separated nanotubes are formed $[7,8]$.

Most commonly CSNs have hexagonal cross sections [6-8], but triangular [9,10] and circular [11] systems have also been achieved. Electrons confined in prismatic CSNs may form conductive channels along the sharp edges [2,12-17]. Rich quantum transport phenomena have been observed in CSNs, e.g., flux periodic, similar to Aharonov-Bohm (AB), magnetoconductance oscillations [6,18], single electron tunneling, or electron interference [6,11]. Very interesting effects have been predicted in the presence of a strong magnetic field perpendicular to the wire axis. In particular, the field induces a complex topology of the electronic states. Low-energy electrons may be found around two channels along the CSN axis where the radial component of the field vanishes by

\footnotetext{
*manoles@ru.is
}

changing sign. Carriers on both sides of the lines are deflected towards opposite directions and thus confined into so-called snaking states [19-22]. Higher-energy electrons start to occupy Landau states and form cyclotron orbits localized in the areas where the radial component of the field takes maximal values. With increasing energy electrons move towards the sample ends and form edge states [22]. To the best of our knowledge experimental investigation of magnetotransport in ballistic CSNs with magnetic field transversal to the nanowire, and of the effects of snaking states, have only recently been attempted [23].

In this paper we focus on thin cylindrical conductive shells since in such systems carrier localization or conductive channels are induced only by an external magnetic field and thus such samples allow us to observe purely magnetic effects. According to our recent calculations the existence of snaking states leads to resolved resonances of the conductance when the CSN is weakly coupled to external leads [24]. In the present paper we extend these results and analyze signatures of snaking states in the conductance for a wide range of sample-lead coupling strength which can be controlled by variable potential barriers. We show that interference of the snaking states, due to backscattering from magnetic or potential barriers, may lead to flux periodic magnetoconductance oscillations, detectable in transport experiments.

The paper is organized as follows. We define the model of the system in Sec. II and describe the computational method in Sec. III. The results and their discussion are presented in Sec. IV while the final remarks are contained in Sec. V.

\section{THE MODEL}

The model of a CSN used here is a simple cylindrical surface of radius $\rho$ and length $L_{z}$, through which a current can flow from one end to another due to a potential bias. We treat the electrons like a cylindrical two-dimensional electron gas. We consider a magnetic field perpendicular to the axis of 

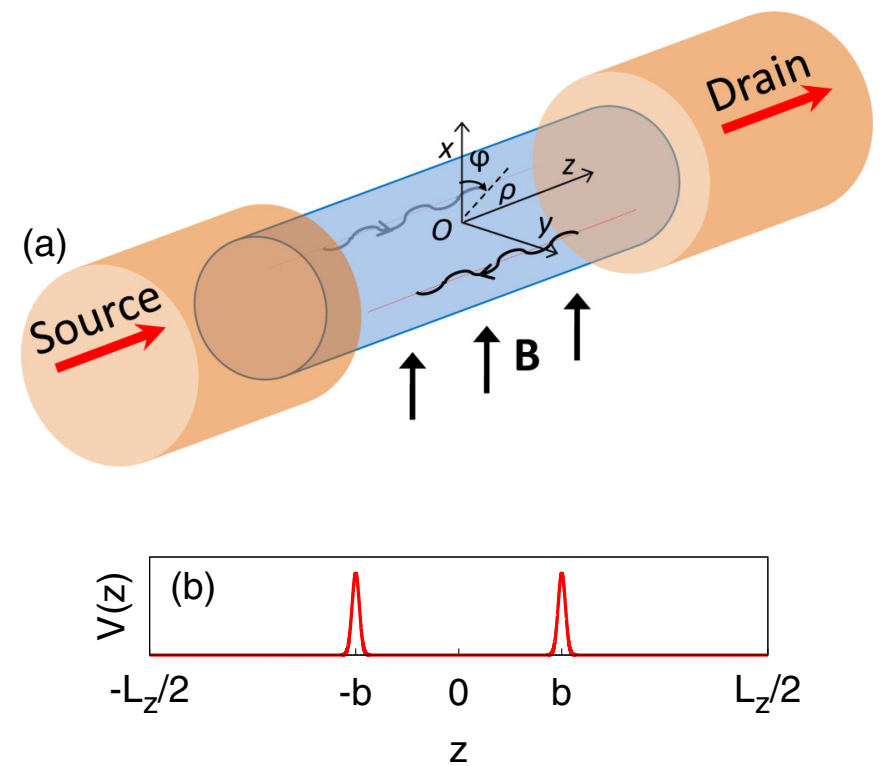

FIG. 1. (a) The CSN is a cylindrical surface of radius $\rho$ and length $L_{z} \mathrm{~nm}$ (blue color). The wavy lines with arrows indicate snaking orbits propagating along the axes of zero radial magnetic field (thin red dotted lines). The contacts with the source and the drain electrodes are shown (gold color). (b) Gaussian potential barriers of variable height placed along the CSN.

the cylinder. When the field is sufficiently strong low-energy electrons can travel along the snaking orbits created along the two lines of the zero radial component, at polar angles $\varphi=\pi / 2$ and $3 \pi / 2$, as illustrated in Fig. 1(a). Two leads are attached to the CSN, one at each end of it, which are treated as particle reservoirs, without a specified shape. Conventionally, we call them Source (S) and Drain (D).

Two potential barriers are placed along the nanowire, at $z=$ $\pm b$, symmetrically around the center $z=0$. The barriers are defined as Gaussian functions $V(z)=V_{0} \exp \left\{-[(z-b) / c]^{2}\right\}$ with a width parameter $c$, and height $V_{0}$, Fig. 1(b). The potential barriers are independent on the polar angle $\varphi$. Their role is to control the contact strength, if placed at the contacts between the nanowire and the leads, and/or contribute to the backscattering of the wave functions.

\section{THE COMPUTATIONAL METHOD}

In order to calculate the conductance of the open cylinder, i.e., the CSN in contact with the external leads, we use the scattering formalism based on the $R$-matrix method. This method has been used in similar transport problems in quantum dots connected to the leads via quantum point contacts [25], in planar nanotransistors [26,27], or in cylindrical bulk nanowires with radial constrictions, at zero magnetic field [28-31]. The approach consists of two parts. In the first part the wave function of an electron at a given energy $E$ is built, both in the leads and in the central scattering region (here the CSN), and matched by continuity conditions at the junctions. In the second part one obtains the $S$ matrix, the transmission function, and the conductance.
We assume that in the leads, close to the junctions with the $\mathrm{CSN}$, the wave function can be written as a combination of plane waves and orbital states:

$$
\psi_{l}(\mathbf{r})=\sum_{m \sigma}\left[\psi_{m \sigma l}^{\text {in }} e^{-i k_{m}\left(z-z_{l}\right)}+\psi_{m \sigma l}^{\text {out }} e^{i k_{m}\left(z-z_{l}\right)}\right] u_{m}(\varphi)|\sigma\rangle,
$$

where $l=S, D$ is a label for the two leads, $z_{l}=\mp L_{z} / 2$ denote the coordinates of the junctions, $\sigma$ is the spin label, and $u_{m}(\varphi)=e^{i m \varphi} / \sqrt{2 \pi}$ are the eigenvectors of the angular momentum, with $m=0, \pm 1, \pm 2, \ldots$. The wave vector $k_{m}$ corresponds to the longitudinal motion of a particular circular mode $m$, being determined by the energy of the incoming electron:

$$
E=\frac{\hbar^{2}}{2 m_{\mathrm{eff}}}\left(k_{m}^{2}+\frac{m^{2}}{\rho^{2}}\right), \quad k_{m}=\sqrt{\frac{2 E m_{\mathrm{eff}}}{\hbar^{2}}-\frac{m^{2}}{\rho^{2}}},
$$

where $m_{\text {eff }}$ is the effective mass of the material. Note that at a fixed energy, depending on $m, k_{m}$ can be real or imaginary. The real values describe open channels, propagating from one lead to another lead, whereas the imaginary values describe closed (or evanescent) channels which are states bound around the scattering region [17].

Although we are formally treating the leads as semi-infinite extensions of the CSN, with the same circular symmetry, in fact the wave functions (1) are important only at (or close to) the boundaries $z_{l}$. Therefore, in principle, the shape of the leads can be arbitrary. In experimental setups the leads are usually perpendicular "finger" electrodes attached to the nanowire sample [18]. Moreover the magnetic field has completely different effects in the leads and in the measured sample. This also motivates us to neglect in our model the magnetic field in the leads. In addition to simplicity, this assumption is helpful to define the contact between the leads and the CSN not only as a mathematical boundary but also as a magnetic barrier. Then, by using the potential barriers well inside the CSN we can also simulate new boundaries of the scattering area, this time with a continuous magnetic field.

In order to calculate the wave function in the CSN region one has to find the eigenstates of the Wigner-Eisenbud (WE) Hamiltonian $\tilde{H}$, satisfying Neumann boundary conditions at the points $z_{l}$ (instead of the Dirichlet conditions familiar for hard wall boundaries):

$$
\tilde{H} \chi_{a}=\epsilon_{a} \chi_{a},
$$

where $a=1,2, \ldots$ is a generic quantum number labeling the WE energies $\epsilon_{a}$ in increasing order. The WE eigenstates $\chi_{a} \equiv|a\rangle$ are expanded in a basis set $|q\rangle=u_{m}(\varphi) u_{n}(z)|\sigma\rangle$, with $u_{n}(z)=A_{n} \cos \left[n \pi\left(z / L_{z}+1 / 2\right)\right], n=0,1,2, \ldots$, and normalization factor $A_{0}=\sqrt{1 / L_{z}}$ and $A_{n}=\sqrt{2 / L_{z}}$ for $n>0$.

The Hamiltonian $\tilde{H}$ is formally built as a regular Hamiltonian $H$, with the kinetic term containing the modified momentum operator $p_{z}+e A_{z}=p_{z}+e B \rho \sin \varphi$, the vector potential being defined in the Landau gauge $\mathbf{A}=(0,0, B y)$, and the Zeeman term depending on the effective $g$ factor of the material:

$$
H=-\frac{\hbar^{2}}{2 m_{\mathrm{eff}} \rho^{2}} \frac{\partial^{2}}{\partial \varphi^{2}}+\frac{\left(p_{z}+e B \rho \sin \varphi\right)^{2}}{2 m_{\mathrm{eff}}}-\frac{1}{2} g_{\mathrm{eff}} \mu_{B} B \sigma_{x} .
$$


With Neumann boundary conditions, implemented via the cosine functions of the basis $|q\rangle$, the resulting linear term in $p_{z}$ is not Hermitian. Therefore the matrix elements of the WE Hamiltonian are defined as $\tilde{H}_{q q^{\prime}}=\left(H_{q q^{\prime}}+H_{q^{\prime} q}^{*}\right) / 2$. This procedure is equivalent to correcting the momentum $p_{z}$ with a surface Bloch operator $L=-i \hbar\left[\delta\left(z-z_{D}\right)-\delta\left(z-z_{S}\right)\right] / 2$ as discussed by other authors [32,33].

The wave function in the CSN can be written as a superposition of WE eigenstates,

$$
\psi(\mathbf{r}, E)=\sum_{a} \alpha_{a}(E) \chi_{a}(\mathbf{r}),
$$

and the coefficients $\alpha_{a}$ are determined by the continuity conditions of the wave functions and their first derivatives at the $z_{l}$ boundaries. Detailed calculations can be found in Appendix A of Ref. [26]. By introducing one more composite label $|\nu\rangle=|m \sigma l\rangle$ the amplitudes of the wave function in the leads, Eq. (1), can be seen as the vectors $\psi^{\text {in }} \equiv\left\{\psi_{v}^{\text {in }}\right\}$ and $\psi^{\text {out }} \equiv\left\{\psi_{v}^{\text {out }}\right\}$, which are related via the continuity conditions, as

$$
\psi^{\text {in }}+\psi^{\text {out }}=-i R K\left(\psi^{\text {in }}-\psi^{\text {out }}\right) .
$$

In Eq. (4) we have introduced two matrices, the matrix of wave vectors with elements $K_{v v^{\prime}}=k_{m} \delta_{v v^{\prime}}$, and the so-called $R$ matrix defined as

$$
R_{\nu \nu^{\prime}}(E)=-\frac{\hbar^{2}}{2 m_{\mathrm{eff}}} \sum_{a} \frac{\langle\nu \mid a\rangle\left\langle\nu^{\prime} \mid a\right\rangle^{\dagger}}{E-\epsilon_{a}} .
$$

The notation $\langle v \mid a\rangle$ stands for the scalar product of the orbital and spin states incorporated in each factor, at the two frontiers $z_{l}$, i.e.,

$$
\langle\nu \mid a\rangle=\langle\sigma| \int_{0}^{2 \pi} u_{m}^{*}(\varphi) \chi_{a}\left(\varphi, z_{l}\right) d \varphi .
$$

The scattering problem is solved by calculating the $S$ matrix, which transforms the "in" states in "out" states,

$$
\psi^{\text {out }}=S \psi^{\text {in }},
$$

and using Eq. (4) it is obtained as

$$
S=-(1-i R K)^{-1}(1+i R K) .
$$

Having the $S$ matrix one can calculate the transmission matrix between the open channels $v$ and $v^{\prime}$

$$
T_{\nu v^{\prime}}(E)=\left|\left(K^{1 / 2} S K^{-1 / 2}\right)_{\nu v^{\prime}}\right|^{2},
$$

and finally the conductance $G$, by summing all contributions from separate leads, i.e., $|v\rangle=|m \sigma S\rangle$ and $\left|v^{\prime}\right\rangle=\left|m^{\prime} \sigma^{\prime} D\right\rangle$ :

$$
G=\frac{e^{2}}{h} \int d E\left(-\frac{\partial \mathcal{F}}{\partial E}\right) \sum_{\substack{m \sigma \\ m \sigma^{\prime}}} T_{m \sigma S, m^{\prime} \sigma^{\prime} D}(E),
$$

where $\mathcal{F}$ denotes the Fermi function.

\section{RESULTS AND DISCUSSION}

In the numerical calculations we used material parameters of InAs, $m_{\text {eff }}=0.023$ and $g_{\text {eff }}=-14.9$. The radius of the CSN was fixed to $\rho=30 \mathrm{~nm}$ and length was $L_{z}=300$ or $2000 \mathrm{~nm}$. The results were convergent in a basis $|q\rangle$ truncated to orbital momenta with $|m| \leqslant 10$ and longitudinal modes $n \leqslant 130$. All channels, both open and closed, were used to calculate the $R$ and $S$ matrices. The temperature was fixed to $T=0.5 \mathrm{~K}$.

\section{A. Zero magnetic field}

First we show in Fig. 2(a) the conductance at zero magnetic field as a function of the chemical potential $\mu$ for several heights of the potential barriers. In this case $L_{z}=300 \mathrm{~nm}$, and the barriers are situated very close to the contacts, at $b= \pm 147.5 \mathrm{~nm}$, having a width of $c=2.5 \mathrm{~nm}$. The results are as expected for ballistic transport in a quantum wire. Without the barriers $\left(V_{0}=0\right)$ the conductance has the familiar steps given by the number of open channels, which is the number of $m$ values yielding a real wave vector $k_{m}$ for the energy $E=\mu$ in Eq. (2), multiplied by the two spin states $\sigma= \pm 1$. By increasing the height of the barriers the conductance drops, because the energy of the electronic states within the CSN increases, and thus a smaller number of open channels remains available up to the fixed Fermi level. Also the conductance begins to oscillate, as a result of the (Fabry-Perot) interference between transmitted and reflected waves, and evolve towards resonances with peaks indicating the density of states in the scattering region. This is a consequence of the fact that the coupling between the scattering region and the leads decreases.
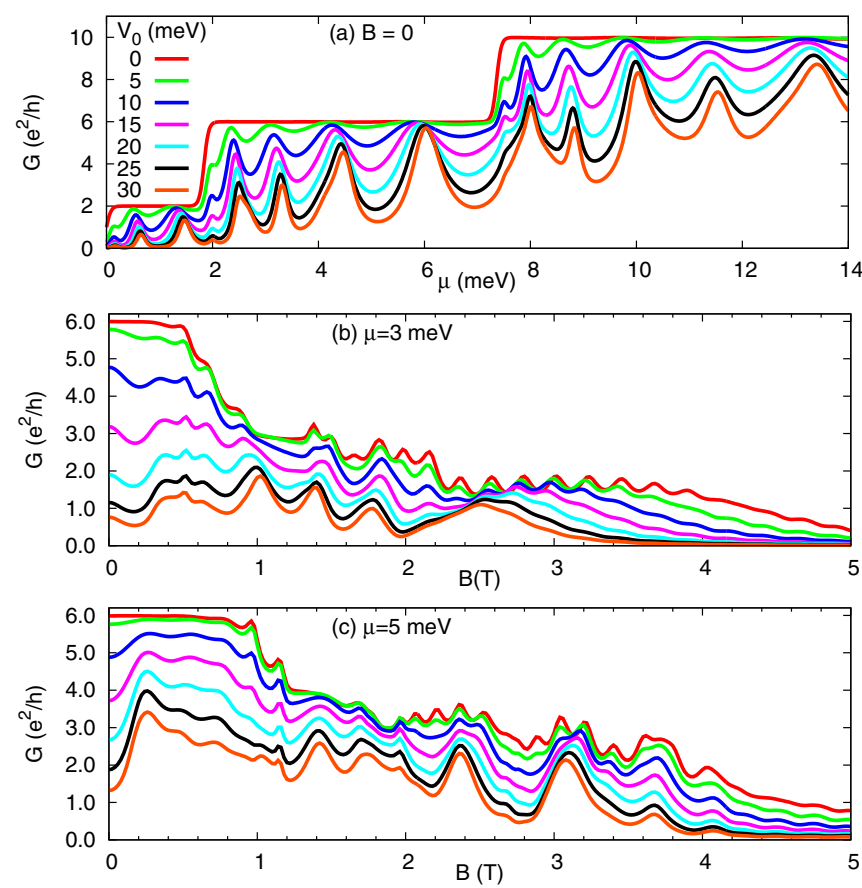

FIG. 2. (a) Conductance vs chemical potential $\mu$ without magnetic field, for contact barriers of height $V_{0}=0,5, \ldots, 30 \mathrm{meV}$, and then vs magnetic field perpendicular to the nanowire, with (b) $\mu=3 \mathrm{meV}$ and (c) $\mu=5 \mathrm{meV}$. The nanowire length is $L_{z}=300 \mathrm{~nm}$ and the barriers are close to the contacts, at $b= \pm 147.5 \mathrm{~nm}$, of width $c=2.5 \mathrm{~nm}$. 


\section{B. Magnetic barriers with adjustable contacts}

Another way to create backscattering of the electrons at contacts is to consider a magnetic barrier, i.e., a magnetic field that exists only in the CSN, but not in the leads, as we assumed in Sec. III. In addition, by adding the potential barriers we can modify the contact strength. In Fig. 2(b), we show how the conductance depends on the strengths of the transverse magnetic field and on the coupling to the leads for chemical potential $3 \mathrm{meV}$. We distinguish two regimes, corresponding to low and high potential barriers, respectively. For weak potentials, i.e., $V_{0}$ up to $5 \mathrm{meV}$, regular conductance oscillations are obtained, with periods $\Delta B$ slightly increasing from 0.20 to $0.22 \mathrm{~T}$ in the interval $B=2-4 \mathrm{~T}$. Each of these oscillations nearly corresponds to a gain of one flux unit $\Phi_{0}=h / e$ through the area of the cylinder projected on the $y z$ plane, $A=2 \rho L_{z}$. According to this estimation the period should be $\Delta B=\Phi_{0} / A=0.23 \mathrm{~T}$. Therefore these oscillations can be considered a kind of $\mathrm{AB}$ interference of snaking states propagating on the lateral sides of the cylinder.

By increasing the height of the potential barriers the AB-like oscillations smear out and the broader conductance peaks emerge. These peaks are produced by the same snaking states, but now as individual resonances occurring in the nearly isolated CSN, only weakly connected to the leads. This case was described by Rosdahl et al. by modeling the contacts with a tunneling parameter [24].

In Fig. 2(c) we show the magnetoconductance with the chemical potential increased to $5 \mathrm{meV}$. The contribution of higher-energy levels results in more complex fluctuations, but the fine structure of the flux periodic oscillations remains visible. The resonances at high potential barriers are now shifted to higher magnetic fields.

\section{WE energy spectra and wave functions}

We can gain more understanding of the $\mathrm{AB}$ oscillations by looking at the WE energy spectra versus magnetic field shown in Fig. 3. In the case of strong coupling between the CSN

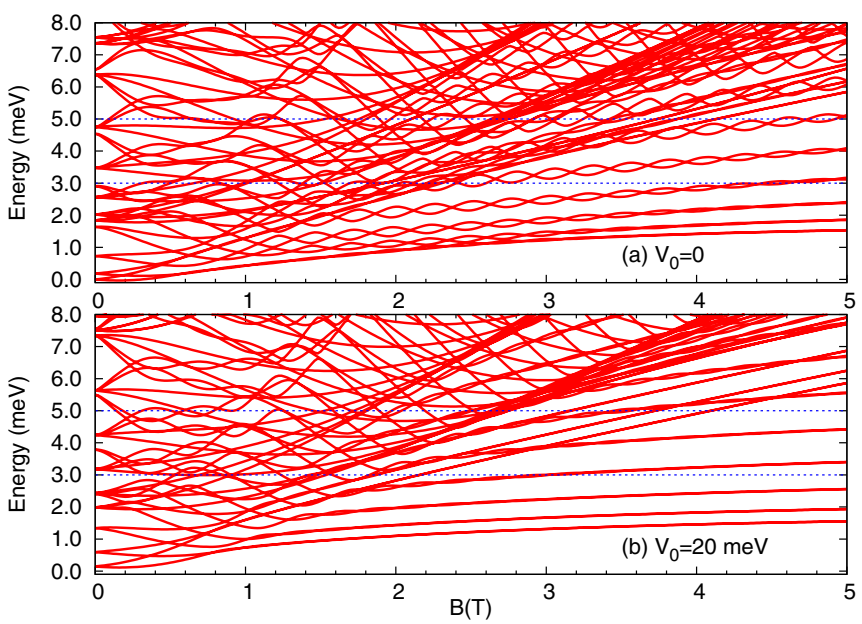

FIG. 3. The Wigner-Eisenbud (WE) energies $\epsilon_{a}$ (a) without contact barriers and (b) with barriers of $V_{0}=20 \mathrm{meV}$. The blue dotted horizontal lines show the chemical potentials used in the magnetoconductance calculations, $\mu=3$ and $5 \mathrm{meV}$. and the leads, e.g., $V_{0}=0$, the low-energy levels form braid shape patterns for $B>1 \mathrm{~T}$, Fig. 3(a). These oscillations affect the conductance, Eq. (6), through the denominators of the $R$ matrix, Eq. (5), which are sensitive to such small changes in the WE energies $\epsilon_{a}$, and induce the $\mathrm{AB}$ oscillations. In fact, the $R$ matrix has a form similar to the Green's functions used by other authors for such scattering-transport calculations [34]. The braids are an indication of snaking states interference in the open CSN. We verified that such an energy spectrum is also obtained for a CSN with a finite thickness of $10 \mathrm{~nm}$, by including in the basis $|q\rangle$ radial wave functions vanishing at the surfaces.

In the presence of high potential barriers the braids shrink and converge towards nearly double degenerate eigenstates, Fig. 3(b), as obtained for the isolated cylinder [24]. The WE spectra can also explain the transition from flux-periodic magnetoconductance to resonant peaks, observed while the height of the potential barriers is being increased. These peaks occur at those magnetic fields for which the snaking states are crossing the Fermi energy.

Further information on the snaking states can be obtained from the wave functions. In the scattering region they are obtained with Eq. (3), using the coefficients $\alpha_{a}$ expressed with the $S$ matrix [28]:

$$
\alpha_{a}(E)=\frac{\hbar^{2}}{2 m_{\mathrm{eff}}} \frac{i}{\sqrt{2 \pi}} \frac{1}{E-\epsilon_{a}} \sum_{\nu}\langle\nu \mid a\rangle^{*} k_{m}\left(1-\sum_{v^{\prime}} S_{\nu v^{\prime}}\right),
$$

where by summing over all labels $v$ we consider electrons incoming from both leads, with wave functions of equal amplitudes. Nevertheless, since the wave functions themselves are not directly involved in the conductance calculations, we look at them only to correlate the behavior of the snaking states in the scattering region with the WE spectrum and the conductance oscillations.

In Fig. 4 we show two distinct situations, for a fixed energy $E=3 \mathrm{meV}$, which is the chemical potential used in Fig. 2(b), and no potential barrier, $V_{0}=0$. For $B=2.91 \mathrm{~T}$ the pair of snaking states have in-phase longitudinal oscillations,

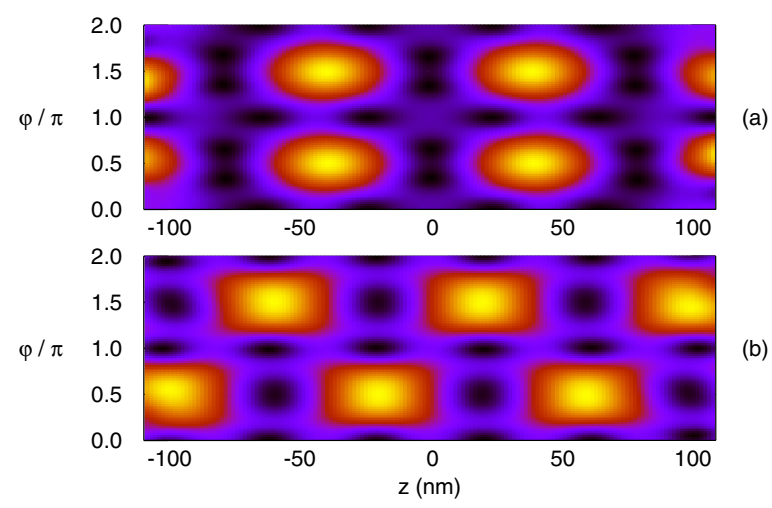

FIG. 4. Probability densities corresponding to the wave functions within the CSN, at energy $E=3 \mathrm{meV}$ and $V_{0}=0$, for (a) $B=2.91 \mathrm{~T}$ when the snaking states are in phase, and (b) $B=3.02 \mathrm{~T}$ when they are in antiphase. Here we show the open cylindrical surface, the vertical axis being the polar angle and the horizontal axis the longitudinal $z$ coordinate. 


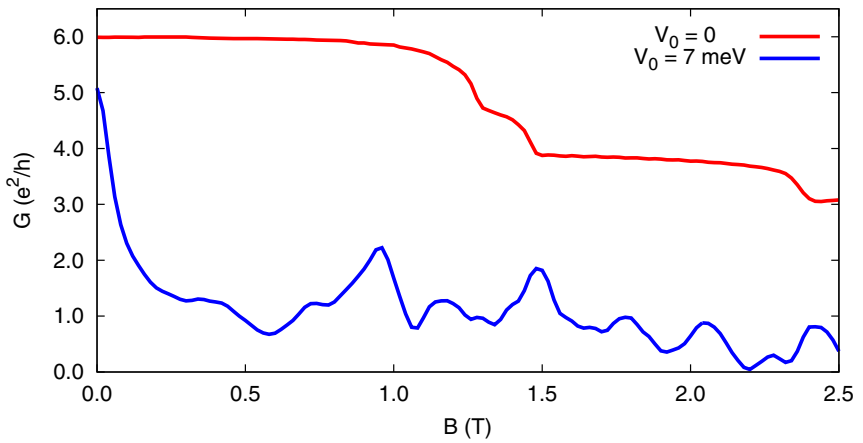

FIG. 5. Conductance vs magnetic field for a nanowire of length $L_{z}=2000 \mathrm{~nm}$. The upper curve was obtained without potential barriers $\left(V_{0}=0\right)$, and the lower curve with two barriers situated at $b= \pm 150 \mathrm{~nm}$, as indicated in Fig. 1(b), of height $V_{0}=7$ $\mathrm{meV}$, and width parameter $c=20 \mathrm{~nm}$. The chemical potential is $\mu=6.5 \mathrm{meV}$.

Fig. 4(a), corresponding to a crossing of the braided WE energies, Fig. 3(a), and to a conductance minimum, Fig. 2(b). For $B=3.02 \mathrm{~T}$ the two snaking states have out-of-phase longitudinal oscillations, Fig. 4(b). In this case the braids are maximally open, and the conductance has a maximum. The out-of-phase structure of the snaking states does not exist in the isolated CSN, but only the in-phase one [24]. Here, for the open CSN, by imposing the potential barriers the lateral shift of the out-of-phase maxima gradually reduces, until they align like in Fig. 4(a). Seen from this angle the braids of the WE spectrum and the $\mathrm{AB}$ oscillations are related to the relative phase of the snaking states.

\section{Potential barriers on a long wire}

In the next example we chose a much longer CSN, of length $L_{z}=2000 \mathrm{~nm}$. The magnetic field still vanishes in the leads, and so there are magnetic barriers at the contacts, which cause backscattering and hence interference. However, the corresponding conductance oscillations are now too dense and too weak to be resolved, because their period is inversely proportional to the wire length. In the absence of any potential barrier the computed magnetoconductance has smooth steps as shown in Fig. 5. The steps reflect the number of propagating (open) channels associated to the complex sub-band structure of the energy spectra of an infinite hollow cylinder in transverse magnetic field [19,20,22,34]. A detailed analysis of these steps is nevertheless beyond the aim of the present paper.
With potential barriers situated at $\pm 150 \mathrm{~nm}$ from the center of the nanowire we can obtain again the flux periodic oscillations, comparable to the ones for the nanowire of $300-n m$ length. The oscillations are now less regular than before, but with an average period of $0.20-0.22 \mathrm{~T}$ within the interval 0.4-2.4 T. In order to reduce the transparency of the barriers and to increase the backscattering we used wider barriers than before, with $c=20 \mathrm{~nm}$. The length of the scattering zone in between the potential barriers is less sharply defined than in the case of the pure magnetic barriers, and so is the real area of the magnetic flux, compared to the reference cross-section area between the barriers, $A=4 \rho b$. Nevertheless, regardless of these imperfections, the oscillations correspond reasonably well with the expected periodicity $\Delta B=\Phi_{0} / A=0.23 \mathrm{~T}$.

\section{CONCLUSIONS}

In conclusion we showed that the existence of the snaking states in a CSN in a transversal magnetic field can be experimentally observed as flux periodic oscillations of the magnetoconductance, with a short CSN strongly coupled to leads. In this case the snaking states behave like transmitted and reflected waves which interfere at the contacts with the leads. In the limit of weak coupling the snaking states can be seen as individual resonances of the conductance. In our model the contacts are primarily simulated by matching two different types of wave functions, in the presence of a magnetic barrier resulting from neglecting the magnetic field in the leads. In order to further modify the transmission and reflection at the contacts we included potential barriers, which reduced the coupling CSN leads, and thus the amplitude of the transmitted waves. Another way to observe the flux periodic oscillations in a transverse magnetic field, although possibly less regular, may be by creating scattering regions with potential barriers, as produced by finger gates placed over a long nanowire [18].

\section{ACKNOWLEDGMENTS}

This work was financially supported by the research funds of Reykjavik University and of the University of Iceland, and by the Icelandic Research Fund. T.O.R. acknowledges support from a European Research Council Starting Grant. We are thankful to Thomas Schäpers and Sebastian Heedt for very interesting discussions [23].
[1] Solid Surfaces, Interfaces and Thin Films, edited by H. Lüth, 5th ed., Graduate Texts in Physics (Springer-Verlag, Berlin, 2010), pp. XVI and 580.

[2] T. Shi, H. E. Jackson, L. M. Smith, N. Jiang, Q. Gao, H. H. Tan, C. Jagadish, C. Zheng, and J. Etheridge, Nano Lett. 15, 1876 (2015).

[3] M.-E. Pistol and C. E. Pryor, Phys. Rev. B 78, 115319 (2008).

[4] B. M. Wong, F. Léonard, Q. Li, and G. T. Wang, Nano Lett. 11, 3074 (2011).
[5] G. Jacopin, L. Rigutti, S. Bellei, P. Lavenus, F. H. Julien, A. V. Davydov, D. Tsvetkov, K. A. Bertness, N. A. Sanford, J. B. Schlager, and M. Tchernycheva, Nanotechnology 23, 325701 (2012).

[6] C. Blömers, T. Rieger, P. Zellekens, F. Haas, M. I. Lepsa, H. Hardtdegen, Ö. Gül, N. Demarina, D. Grützmacher, H. Lüth, and T. Schäpers, Nanotechnology 24, 035203 (2013).

[7] T. Rieger, M. Luysberg, T. Schäpers, D. Grützmacher, and M. I. Lepsa, Nano Lett. 12, 5559 (2012). 
[8] F. Haas, K. Sladek, A. Winden, M. von der Ahe, T. E. Weirich, T. Rieger, H. Lüth, D. Grützmacher, T. Schäpers, and H. Hardtdegen, Nanotechnology 24, 085603 (2013).

[9] F. Qian, Y. Li, S. Gradeak, D. Wang, C. J. Barrelet, and C. M. Lieber, Nano Lett. 4, 1975 (2004).

[10] F. Qian, S. Gradeak, Y. Li, C.-Y. Wen, and C. M. Lieber, Nano Lett. 5, 2287 (2005).

[11] T. Richter, C. Blömers, H. Lüth, R. Calarco, M. Indlekofer, M. Marso, and T. Schäpers, Nano Lett. 8, 2834 (2008).

[12] J. Jadczak, P. Plochocka, A. Mitioglu, I. Breslavetz, M. Royo, A. Bertoni, G. Goldoni, T. Smolenski, P. Kossacki, A. Kretinin, H. Shtrikman, and D. K. Maude, Nano Lett. 14, 2807 (2014).

[13] A. Bertoni, M. Royo, F. Mahawish, and G. Goldoni, Phys. Rev. B 84, 205323 (2011).

[14] M. Royo, A. Bertoni, and G. Goldoni, Phys. Rev. B 89, 155416 (2014).

[15] M. Royo, C. Segarra, A. Bertoni, G. Goldoni, and J. Planelles, Phys. Rev. B 91, 115440 (2015).

[16] M. Fickenscher, T. Shi, H. E. Jackson, L. M. Smith, J. M. Yarrison-Rice, C. Zheng, P. Miller, J. Etheridge, B. M. Wong, Q. Gao, S. Deshpande, H. H. Tan, and C. Jagadish, Nano Lett. 13, 1016 (2013).

[17] A. Sitek, L. Serra, V. Gudmundsson, and A. Manolescu, Phys. Rev. B 91, 235429 (2015).

[18] O. Gül, N. Demarina, C. Blömers, T. Rieger, H. Lüth, M. I. Lepsa, D. Grützmacher, and T. Schäpers, Phys. Rev. B 89, 045417 (2014).

[19] G. Ferrari, A. Bertoni, G. Goldoni, and E. Molinari, Phys. Rev. B 78, 115326 (2008).
[20] G. Ferrari, G. Goldoni, A. Bertoni, G. Cuoghi, and E. Molinari, Nano Lett. 9, 1631 (2009).

[21] M. Royo, A. Bertoni, and G. Goldoni, Phys. Rev. B 87, 115316 (2013).

[22] A. Manolescu, T. Rosdahl, S. Erlingsson, L. Serra, and V. Gudmundsson, Eur. Phys. J. B 86, 445 (2013).

[23] S. Heedt, W. Prost, J. Schubert, D. Grützmacher, and T. Schäpers, conference presentation given at Nanowires 2014, Eindhoven; (private communication).

[24] T. O. Rosdahl, A. Manolescu, and V. Gudmundsson, Nano Lett. 15, 254 (2015).

[25] E. R. Racec, U. Wulf, and P. N. Racec, Phys. Rev. B 82, 085313 (2010).

[26] G. A. Nemnes, U. Wulf, and P. Racec, J. Appl. Phys. 96, 596 (2004).

[27] G. A. Nemnes, U. Wulf, and P. Racec, J. Appl. Phys. 98, 084308 (2005).

[28] P. N. Racec, E. R. Racec, and H. Neidhardt, Phys. Rev. B 79, 155305 (2009).

[29] P. N. Racec, Phys. Status Solidi B 251, 195 (2014).

[30] G. A. Nemnes, L. Ion, and S. Antohe, J. Appl. Phys. 106, 113714 (2009).

[31] G. A. Nemnes, L. Ion, and S. Antohe, Physica E 42, 1613 (2010).

[32] T. Jayasekera, K. Mullen, and M. A. Morrison, Phys. Rev. B 74, 235308 (2006).

[33] K. Varga, Phys. Rev. B 80, 085102 (2009).

[34] Y. Tserkovnyak and B. I. Halperin, Phys. Rev. B 74, 245327 (2006). 